
\title{
25 Research Soure \\ The expression of cerebrospinal fluid pentraxin-3 in patients with hemorrhagic stroke
}

\section{yin Fu}

Hunan University of Chinese Medicine;The Second People's Hospital of Hunan Province https://orcid.org/0000-0002-3589-2374

\section{Qing Zhu}

Hunan University of Chinese Medicine;The Second Peoples Hospital of Hunan Province

\section{Qi Guo}

Hunan University of Chinese Medicine;The Second Peoples Hospital of Hunan Province Junyu He

Peking University People's Hospital

\section{Guofeng Xu}

Hunan University of Chinese Medicine;The Second Peoples Hospital of Hunan Province

\section{Biqiong Ren}

Hunan University of Chinese Medicine;The Second Peoples Hospital of Hunan Province

Guoying Zou ( $\nabla$ zouwanshan75@163.com )

\section{Research article}

Keywords: stroke, PTX3, Inflammation, prognosis

Posted Date: March 29th, 2019

DOI: https://doi.org/10.21203/rs.2.1718/v1

License: (c) (1) This work is licensed under a Creative Commons Attribution 4.0 International License.

Read Full License 


\section{Abstract}

Background: There exists brain tissue inflammation and endothelial function damage in stroke. Method: We investigated serum and cerebrospinal fluid (CSF) levels of PTX3, HSP70, VEGF, IL-6, CRP in patients with stroke, this study was conducted on 85 standard stroke patients during 2015-2016, include ischemic stroke (IS) group (52 cases) and hemorrhagic stroke (HS) group (33 cases). The patients without inflammatory and neurological disease were the control group (20 cases). The levels of PTX3, HSP70, VEGF, IL-6 and CRP in serum on the 1th, 5th and 14th day after admission were measured and compared them with that in cerebrospinal fluid, NIHSS and Barthel score were also performed for assessing patients with HS. The results showed that the serum levels of PTX3, HSP70, VEGF, IL-6, CRP with HS were higher than that with IS patients $(P<0.001, P=0.16, P=0.08, P=0.12, P<0.001$, respectively), and $P T X 3$ level began to fall after 14th day $(P=0.015)$. The cerebrospinal fluid of PTX3, HSP70,VEGF and IL-6 levels in patients with HS were higher than that of normal control group $(P=0.002, P=0.003, P<0.001, P<0.001$, respectively). The levels of PTX, HSP70, IL-6 in cerebrospinal fluid were higher than that in the serum $(P=0.026, P<0.001, P<0.001$, respectively), and VEGF, CRP instead $(P=0.011, P<0.001$, respectively). The level of PTX3 in cerebrospinal fluid was positively correlated with IL-6 in patients with HS $(P<0.001)$, PTX3 and IL- 6 in patients with HS were positively correlated with patients' NIHSS score $(P=0.007$, $P<0.001$, respectively), and negatively correlated with Barthel index score $(P=0.023, P<0.001$, respectively). Inflammation and endothelial damage were more severe in patients with HS. The levels of PTX3 and IL- 6 in cerebrospinal fluid could assess the severity, progression, and prognosis of HS. PTX3 may be a potential biomarker in HS, it could provide potent basis for doctors to select appropriate treatment measures as soon as possible.

\section{Background}

Cerebral stroke is a common cerebrovascular disease in neurology department. It is the first leading cause of death in China's urban and rural areas, and more than 1 million people died each year. The survivors also suffer from the consequence of hemiplegia and labor loss. Stroke is divided into ischemic stroke (IS) and hemorrhagic stroke (HS). IS also called the cerebral infarction, Ischemic stroke is the most common type of cerebrovascular disease which caused by the cerebral blood supply obstacles, brain tissue necrosis, nerve function defect, accounting for about $75 \%$ of all cerebrovascular disease and less harmful than HS. HS is caused by a variety of factors including cerebral vascular wall lesions, necrosis and rupture hemorrhage, including cerebral hemorrhage and subarachnoid hemorrhage, is characterized by acute onset, high disability and mortality. Its pathologic mechanism is closely related to vascular endothelial injury and inflammatory response. This study is aim to provide the basis for clinical diagnosis, treatment and prognosis by detecting the levels of pentraxin-3(PTX3), Heat shock protein 70 (Hsp70), vascular endothelial growth factor (VEGF), Interleukin-6 (IL-6), c-reactive protein (CRP) in serum and cerebrospinal fluid in patients with HS, and know whether the above indicators associated with inflammation and endothelial function change in HS, and its influence on the occurrence and development of the disease, provide the basis for clinical diagnosis and treatment. 


\section{Materials And Methods}

Research subjects. From August 2015 to August 2016, collecting blood and cerebrospinal fluid samples of patients with stroke in the second people's hospital of Hunan province after the test, which were send to the laboratory for examination in order to consider the illness, the cerebrospinal fluid samples of patients without inflammatory and neurological diseases were selected as the control group, which were also collected for the diagnosis and treatment of the disease at the same period. According to the American NINCDS standards (1978): clinical records of the history of rapid onset and symptoms last more than 24 hours or the limited brain symptoms of leading to death and meeting one of the following: imaging examination have cerebral hemorrhage, or cerebral hemorrhage was confirmed during the surgery, or lumbar puncture cerebrospinal fluid in hemorrhagic (non-invasive). It subarachnoid hemorrhage in accordance with the following two criteria: (1) the clinical records of acute onsets with the following clinical manifestations: severe headache and/or vomiting when awakening for the first time after admission, consciousness levels drop; Brain stimulation; Hemorrhage in the retina (vitreous membrane). Imaging studies have demonstrated hematoma or subarachnoid hematoma in the lateral fissure of the brain, or in the basal pool or in the ventricle. Confirmed with cystic artery aneurysms (2) surgery or arteriovenous malformation, or angiography confirmed have cystic artery aneurysms or abnormal arteriovenous as evidence of bleeding source (such as normal vasospasm or near an aneurysm of a blood clot). The blood and cerebrospinal fluid specimens of $5 \mathrm{~d}$ and $14 \mathrm{~d}$ in HS patients were tracked in time after admission. Screening and tracking a group of 85 cases, in which a group of 52 cases of HS (male 37, female 15 cases), aged $27 \sim 87$, an average of 69 years of age, IS group and 33 patients (male 20 , female 13), aged $44 \sim 87$, the average at the age of 67 . In the control group, 20 patients (13 males and 7 females) were aged from 3 to 80 years, with an average age of 52 (.

Inclusion criteria. All HS patients were able to be traced the blood and cerebrospinal fluid specimens on 5 $\mathrm{d}$ and $14 \mathrm{~d}$ after admission. Exclusion criteria: patients were not given blood and cerebrospinal fluid samples from $5 \mathrm{~d}$ and $14 \mathrm{~d}$ after admission; Statins were taken within 1 month before admission; Patients with ischemic heart and cerebrovascular disease, Tumor, Infectious diseases, the operation performed within 5 days and other serious systemic diseases.

Disease assessment. All the selected HS patients were assessed by the stroke scale of the United States National Institutes of Health Stroke Scale (NIHSS) for the severity of the disease and Barthel index score for prognosis of disease.

Specimen acquisition. Retrospective to return the patients' serum and cerebrospinal fluid samples on $1 \mathrm{~d}$, $5 \mathrm{~d}, 14 \mathrm{~d}$ after admission, these specimens were centrifuged, frozen, and reserved in $-80^{\circ} \mathrm{C}$ until detected.

Measurements. PTX3, HSP70 and VEGF were measured by ELISA with enzyme-linked immunosorbent assay (ELISA). These kits were provided by R\&D Systems and were performed according to the reagent specifications. IL- 6 was determined by electrochemiluminescence method. The kit was from Roche company and the instrument was Cobas e601. CRP was determined by latex enhanced 
immunoturbidimetric assay. The kit was from MeiKang biotechnology company, and the instrument was Siemens ADVIA 2400.

Statistical analysis. SPSS 16.0 software (SPSS Inc., Chicago, USA) was used to statistical analysis, the measurement data in accordance with normal distribution shall be represented by $\pm S D$, one-way ANOVA analysis was used to evaluate the differences between three groups, and independent sample $t$ test was applied for the two groups comparison. Paired $t$ test was used to analyze different samples of the same patient. $P<0.05$ was considered significant. Graphpad Prism 5.0 (Graphpad Software, La Jolla, CA, USA) was used for graph statistics.

\section{Results}

\section{More severe inflammation injury and endothelial function damage in circulating blood in patients with} HS. We detected circulating blood in patients with stroke on admission and found the levels of PTX3, HSP70, VEGF, IL- 6 and CRP in patients with HS were higher than that with IS $(P<0.001, P=0.16, P=0.08$, $P=0.12, P<0.001$, respectively) and the control group $(P=0.018, P=0.043, P=0.008, P=0.026, P=0.004$, respectively), the results showed that there was more serious inflammation injury and endothelial function damage in patients with HS, while Inflammation and endothelial damage were not detected in patients with IS on admission (Table 1).

The changes of inflammation and endothelial function in circulating blood in patients with HS. The above results showed that the HS patients had inflammation and endothelial damage, in order to better understand the status change of patients, we observed the changes of serum PTX3, HSP70, VEGF, IL-6 and VEGF levels in HS patients (on $1 \mathrm{~d}, 5 \mathrm{~d}, 14 \mathrm{~d}$ after admission) to monitor the patients' inflammatory condition and endothelial injury. The results showed that the PTX3 level of HS patients had a downward trend on $14 \mathrm{~d}$ after admission $(P=0.015)$, and no trend change in other parameters (figure 1 ), which indicated only PTX3 could reminder the development of the disease.

The inflammatory status and endothelial function in brain in HS patients. The patients in HS kept in the severe inflammation and vascular endothelial injury in circulating system, then to further know if there were similar changes in HS disease itself--craniocerebral injury, we observed the above test parameters in cerebrospinal fluid, we found the levels of PTX3, HSP70, VEGF and IL-6 in cerebrospinal fluid of HS patients were higher than the control subjects $(P=0.002, P=0.003, P<0.001, P<0.001$, respectively), there were inflammation and vascular endothelial injury in brain (figure 2).

The regulation of neurological inflammation and vascular endothelial factors in HS patients. As a result, so far, we found inflammation and vascular endothelial damage in both the brain and circulatory system in HS patients. However, we wondered where these increase of inflammation and endothelial factors come from. So we compared these parameters in the cerebrospinal fluid with those in blood (figure 3), the results manifested that the levels of PTX3, HSP70 and IL-6 in cerebrospinal fluid were higher than those in serum $(P=0.026, P<0.001, P<0.001$, respectively), while the levels of VEGF and CRP were lower than 
those in serum $(P=0.011, P<0.001$, respectively), which indicated that the changes of inflammation and vascular endothelial factors in HS patients were related to neurological damage.

The correlation analysis of PTX3 in cerebrospinal fluid. After the admission of HS patients, PTX3 showed a downward trend in circulating blood on $14 \mathrm{~d}$, which indicated PTX3 change as the disease progresses, and the change of PTX3 in cerebrospinal fluid was more significant. We conducted the correlation analysis of PTX3 in cerebrospinal fluid (Table 2, Figure 4), the results showed that the levels of PTX3 and IL-6 in cerebrospinal fluid of HS patients were positively correlated $(r=0.631, P<0.001)$, and these two were also positively correlated with NIHSS score $(r=0.412, P=0.007)$, while negatively correlated with Barthel index score $(r=0.608, P<0.001)$, the levels of PTX3 and IL- 6 in cerebrospinal fluid could assess the severity and prognosis of the disease.

Therefore, in patients with HS, the expressions of PTX3, HSP70, VEGF and IL-6 in cerebrospinal fluid were up-regulated on admission, then by respective mechanisms, they reduced neurological injury, broke through the broken BBB into circulating blood, and stimulated the release of various factors, such as CRP, to reduce inflammation and protect the nerve cells (Figure 5), the levels of PTX3 in cerebrospinal fluid could assess the severity, progression, and prognosis of the disease. PTX3 may be a potential biomarker in HS.

\section{Discussion And Conclusions}

HS is a kind of abrupt onset and extremely dangerous stroke disease. HS has the characteristics of high mortality and poor prognosis, the pathological mechanism is closely related to brain edema formation, hematoma expansion and damage, thrombin release, inflammatory reaction and so on. A large number of studies have shown that the inflammatory mediators could destroy the blood-brain barrier (BBB) after cerebral hemorrhage, directly damage brain tissue and aggravate the disease. The level of interleukin increased in HS patients, which blocked neuroprotective activation effects, and positively correlated with the disease severity [1-2]. The expressions of tumor necrosis factor-a, monocyte chemotactic protein-1 and intercellular adhesion molecule- 1 increased significantly in cerebral hemorrhage after $24 \mathrm{~h}$, and the inhibition of the these factors expression could alleviate brain tissue damage [3]. The HS pathogenesis is closely related to vascular endothelial injury and inflammatory response. Various related indicators have respective specific changes during the disease development, which provides a strong basis for the diagnosis and treatment of the disease.

PTX3 is a member of the pentraxin super family, an acute phase reaction product, and mainly comes from the heart and blood vessels tissue damage. PTX 3 is considered to be a marker of inflammation. Compared with c-reactive protein derived from the liver, PTX3 is more sensitive and more specific in predicting tissue injury. Most studies have shown that PTX 3 was closely associated with cardiovascular disease [4-5], pathological observation suggested that PTX3 could be generated by macrophages in atherosclerotic plaques. PTX3 directly reflected the local inflammation state of coronary atherosclerotic plaque and plaque instability, and was related to the stenosis degree of coronary artery [6-7]. Studies on nervous system diseases have shown PTX3 was positively correlated with the disease severity and poor 
prognosis in IS, and predicted the mortality [8]. We monitored the levels of serum inflammatory factors and vascular endothelial growth factors in patients with HS, and found that the expression of PTX3 in circulating blood increased (Table 1). PTX3 began to decline with the disease improvement after admission 14 days (Figure 1). The expression of PTX 3 in cerebrospinal fluid also increased (Figure 2), and its concentration was higher than that in circulating blood (Figure 3). We suggested that the level of PTX3 in cerebrospinal fluid elevated due to neurological damage, and then released into blood by damage vascular endothelial. Studies confirmed PTX 3 knockout affected the recovery of BBB and cerebral edema in cerebrovascular disease, because astrocyte-derived PTX3 protected BBB integrity under ischemic states, exogenous PTX3 significantly increased expression of ZO-1 and Claudin 5, enhanced endothelial barrier tightness, PTX3 may negatively regulate VEGF actions on cerebral endothelial cell permeability by directly binding and capturing VEGF, which suggested that PTX3 could adjust nerve and vascular regeneration and have repair function[9-11]. So elevated PTX3 could protect the nerve function and repair damaged brain tissue in patients with HS.

Hsp70, a inflammatory factor, is a structurally highly conserved polypeptide. Hsp70 could protect and promote cells to repair damage caused by various stimuli. In IS animal models, studies have shown that Hsp70 resisted against multiple types of cell death, including apoptosis, necrosis, and other cell death pathways [12]. Our study found that the level of Hsp70 increased in patients with HS (Table 1, Figure 2), further, the expression of Hsp70 in cerebrospinal fluid was higher than that in circulating blood (Figure 3). Hsp70 could inhibit cytochrome $c$ release in experimental stroke models and block apoptosis inducing factor (AIF) translocation to the brain barrier, which influenced cell death pathway, reduced ischemic brain injury, and improved nervous system prognosis [13-14]. We suggested that Hsp70 came from the inflammation of the nervous system in HS. Under the adverse conditions of stress, brain tissues could increase the expression of Hsp70 through self-regulation, so as to improve the resistance and protect nerve cells.

IL-6 is a kind of pleiotropic cytokine with biological activity. IL-6, secreted by T cells, B cells, mononuclear macrophages and endothelial cells after stimulated by inflammation, can induce hepatocyte to synthesize acute reactive protein (such as c-reactive protein) and participate in the inflammatory response. CRP, an acute reactive protein, is a part of the non-specific immune mechanism, and may bind to platelet activating factor (PAF) to reduce inflammation. The expression of IL-6 was up-regulated in astrocytes and microglia with acute ischemia, which may have neurotoxic and neuroprotective dual effects [15]. We detected the levels of IL-6 and CRP were increased in patients with stroke, and were higher in patients with HS than with IS (Table 1). The level of serum IL-6 reflected the severity of the stroke, and may be a biomarker of acute cerebral injury [16]. Our results coincided with the severity what was often more serious in HS than in IS. In HS, the nerve cells were induced to synthesize IL-6 to promote the growth of neurons.

VEGF, a vascular endothelial marker, is a specific heparin-binding growth factor. Under physiological conditions, VEGF and its receptor maintain a low levels, and are up-regulated when vascular endothelial cells proliferate. In the central nervous system, VEGF was regulated by factors such as inflammation and 
hypoxia, which promoted endothelial cell proliferation and induced new angiogenesis [17-18]. There is more VEGF researches on ischemic brain disease. Most studies have shown that VEGF involved in various periods of IS disease, and mediated neural injury and inflammation in the early stage, broke down BBB integrity by down-regulating protein and mRNA levels of claudin 5 and occluding [19]. While VEGF could improve circulation, inhibit apoptosis and protect nerve in ischemic cerebrovascular diseases [20]. We found that the level of VEGF was significantly increased in patients with HS (Table 1, Figure 2), however, the level of VEGF in cerebrospinal fluid was lower than that in serum (Figure 3), which revealed that there was decreased vascular endothelial function in brain tissue during $\mathrm{HS}$, and neuroprotective function was damaged.

Our results showed that there were inflammation and endothelial function damage in HS, and PTX3 was closely related to the development of HS. The levels of PTX3, IL-6, HSP70 and VEGF in patients with HS was higher than that with IS (Table 1), which disclosed there were more serious damage in patients with HS than that in IS, and these were related to high mortality, disability and poor prognosis. Meanwhile, increased levels of PTX3 and VEGF also supported the view that PTX3 regulated angiogenesis in stroke patients. To clarify the main sources of inflammation and injury, we compared relevant markers of serum with that of cerebrospinal fluid in patients with HS, and found that the levels of PTX3 and IL-6 in cerebrospinal fluid were higher, which suggested that they were products of neuroinflammatory injury, and the serum expression was increased attribute to the destruction of BBB. The expression of IL- 6 upregulated in cerebrospinal fluid under stress conditions. IL-6 induced the synthesis of CRP in liver cells after entering circulating blood through the damaged BBB, and then reduced the inflammatory response to protect nerve cells (Figure 5). In animal experiments, circulating injection of IL-1 could enhance almost double the concentration of plasma PTX3 in mice without brain injury, but could not affect the expression of PTX3 in cerebral cortex and striatum, which showed that PTX3 in circulating blood could not pass through the BBB [10]. In patients with subarachnoid hemorrhage, Zanier ER team [21] also proposed that PTX3 comes from the disease itself, and that PTX 3 in cerebrospinal fluid involved vasospasm. Our results also showed that ten level of PTX3 was higher in cerebrospinal fluid than that in serum, which revealed PTX3 increase ascribed to HS disease itself. The level of serum PTX3 showed a significant downward trend until 14 days after admission in patients with HS, which indicated the good prognosis of the disease. In PTX3 related analysis in cerebrospinal fluid in patients with HS, PTX3 was positively related with IL-6, both PTX3 and IL-6 were positively related with patients' NIHSS score, while both were negatively related with the patients' Barthel index score. The above all of these suggested that PTX3 is associated with the nervous system damage in patients with HS, the higher the levels of IL-6 and PTX3, the more severe the neurological damage, and the poorer the ability of daily living, and so IL- 6 and PTX3 were adverse prognostic factors. The research of Wi-sun Ryu group [22] were consistent with our study, increased PTX3 level was independently associated with disease mortality in IS, the combination detection of PTX3 and NIHSS had better ability of predict mortality than that of single.

In Conclusion, the levels of PTX3, HSP70, VEGF, IL- 6 and CRP in serum and cerebrospinal fluid increased in patients with HS, PTX3, IL-6 and Hsp70 were secreted due to the inflammation of the nervous system itself, then released to circulating blood through damaged BBB, and the levels of PTX3 and IL- 6 in 
cerebrospinal fluid could assess the severity, progression, and prognosis of the disease. PTX3 may be a potential biomarker in HS, it could provide potent basis for doctors to select appropriate treatment measures as soon as possible.

\section{Abbreviations}

ischemic stroke (IS)

hemorrhagic stroke (HS)

pentraxin-3(PTX3)

heat shock protein 70 (Hsp70)

vascular endothelial growth factor (VEGF)

interleukin-6 (IL-6)

c-reactive protein (CRP)

enzyme-linked immunosorbent assay (ELISA)

United States National Institutes of Health Stroke Scale (NIHSS)

apoptosis inducing factor (AIF)

platelet activating factor (PAF)

\section{Declarations}

Ethics approval and consent to participate: The experimental protocol was established, according to the ethical guidelines of the Helsinki Declaration and was approved by the Human Ethics Committee at Brain hospital of hunan province. Written informed consent was obtained from individual or guardian participants (Included participants under the age of 16).

Consent for publication: Not applicable

Competing interests: The authors declare that they have no competing interests.

Availability of data and material: Not applicable

Funding: This study was supported by grants from the Hunan Natural Science Foundation (2018JJ6102) and the Health and Family Planning Commission of Hunan Province plan projects (B2014-103). 
Authors' contributions: Guoying Zou and Yin Fu carried out the concepts, design, definition of intellectual content, literature search, data acquisition, data analysis and manuscript editing. Qing Zhu, Qi Guo, Junyu He and Guofeng Xu provided assistance for samples acquisition and detection, data analysis and statistical analysis. Biqiong Ren and Guoying Zou performed manuscript review. Yin Fu drafted the manuscript. All authors have read and approved the content of the manuscript.

\section{References}

1. Tu CJ, Liu WG, Dong XQ, et al. Association of interleukin-11 with mortality in patients with spontaneous basal ganglia haemorrhage[J]. Journal of International Medical Research, 2011, 39(4):1265-1274.

2. Wang K W, Cho C L, Chen $\mathrm{H} \mathrm{J}$, et al. Molecular biomarker of inflammatory response is associated with rebleeding in spontaneous intracerebral hemorrhage[J]. European Neurology, 2011, 66(6):322-327.

3. Ma Q, Manaenko A, Khatibi N H, et al. Vascular adhesion protein-1 inhibition provides antiinflammatory protection after an intracerebral hemorrhagic stroke in mice[J]. Journal of Cerebral Blood Flow \& Metabolism Official Journal of the International Society of Cerebral Blood Flow \& Metabolism, 2011, 31(3):881-893.

4. Guo TM, Huang LL, Liu K, et al. Pentraxin 3 (PTX3) promoter methylation associated with PTX3 plasma levels and neutrophil to lymphocyte ratio in coronary artery disease[J]. Geriatr Cardiol, 2016,13(8), 712-717.

5. Carrizzo A, Lenzi P, Procaccini C, et al. Pentraxin 3 Induces Vascular Endothelial Dysfunction Through a P-selectin/Matrix Metalloproteinase-1 Pathway[J]. Circulation, 2015, 131(17), 1495-1505.

6. Kimura S, Inagaki H, Haraguchi G, et al. Relationships of Elevated Systemic Pentraxin-3 Levels With High-Risk Coronary Plaque Components and Impaired Myocardial Perfusion After Percutaneous Coronary Intervention in Patients With ST-Elevation Acute Myocardial Infarction[J]. Circulation Journal Official Journal of the Japanese Circulation Society, 2013, 78(1):159-169.

7. Guo T, Huang L, Liu C, et al. The clinical value of inflammatory biomarkers in coronary artery disease: PTX3 as a new inflammatory marker[J]. Exp Gerontol, 2017, 97:64-67.

8. Qin LZ, Li W, Huang Y, et al. PTX3 expression in the plasma of elderly ACl patients and its relationship with severity and prognosis of the disease[J]. Eur Rev Med Pharmacol Sci, 2016, 20(19):4112-4118.

9. Rodriguez-Grande B, Varghese L, Molina-Holgado F, et al. Pentraxin 3 mediates neurogenesis and angiogenesis after cerebral ischaemia[J]. J Neuroinflammation, 2015, 12:15.

10. Rodriguez-Grande B, Swana M, Nguyen L, et al.The acute-phase protein PTX3 is an essential mediator of glial scar formation and resolution of brain edema after ischemic injury[J]. J Cereb Blood Flow Metab, 2014, 34(3):480-488. 
11. Shindo A, Maki T, Mandeville ET, et al. Astrocyte-Derived Pentraxin 3 Supports Blood-Brain Barrier Integrity Under Acute Phase of Stroke[J]. Stroke, 2016, 47(4):1094-1100.

12. De Maio A. Extracellular Hsp70: export and function.[J].Current Protein \& Peptide Science, 2014, 15(3):225- 231.

13. Kim JY, Han Y, Lee JE, et al. The 70-kDa heat shock protein (Hsp70) as a therapeutic target for stroke[J]. Expert Opin Ther Targets, 2018, 22(3): 191-199.

14. Kim JY, Yenari MA. The immune modulating properties of the heat shock proteins after brain injury[J]. Anat Cell Biol, 2013, 46(1):1-7 .

15. Bustamante A, Sobrino T, Giralt D, et al. Prognostic value of blood interleukin-6 in the prediction of functional outcome after stroke: A systematic review and meta-analysis[J]. Journal of Neuroimmunology, 2014, 274(1-2):215-224.

16. Whiteley W, Wardlaw J, Dennis M, et al. The use of blood biomarkers to predict poor outcome after acute transient ischemic attack or ischemic stroke[J]. Stroke, 2012, 43(1):86-91.

17. Libby P, Ridker P M, Hansson G K. Progress and challenges in translating the biology of atherosclerosis[J]. Nature, 2011, 473(7347):317-325.

18. Li N, Wang P, Ma X L, et al. Effect of bone marrow stromal cell transplantation on neurologic function and expression of VEGF in rats with focal cerebral ischemia[J]. Molecular Medicine Reports, 2014, 10(5):2299-2305.

19. Argaw AT, Gurfein BT, Zhang Y, et al. Vegf-mediated disruption of endothelial cln-5 promotes bloodbrain barrier breakdown[J]. Proc Natl Acad Sci USA. 2009,106(6):1977-1982.

20. Chen C, Ostrowski R P, Zhou C, et al. Suppression of hypoxia-inducible factor-1alpha and its downstream genes reduces acute hyperglycemia-enhanced hemorrhagic transformation in a rat model of cerebral ischemia[J]. Journal of Neuroscience Research, 2010, 88(9):2046-2055.

21. Zanier ER, Brandi G, Peri GC, et al. Cerebrospinal fluid pentraxin 3 early after subarachnoid hemorrhage is associated with vasospasm[J]. Intensive Care Med, 2011, 37(2):302-309.

22. Ryu W-S, Kim CK, Kim BJ, et al. Yoon BW. Pentraxin 3: a novel and independent prognostic marker in ischemic stroke[J]. Atherosclerosis, 2012, 220(2):581-586.

\section{Tables}

Table 1 The inflammation state and endothelial function in patients with stroke 


\begin{tabular}{|c|c|c|c|c|c|c|}
\hline Groups & $\mathrm{n}$ & $\begin{array}{l}\text { РTX3 } \\
(\mathrm{ng} / \mathrm{ml})\end{array}$ & $\begin{array}{l}\text { HSP70 } \\
(\mathrm{ng} / \mathrm{ml})\end{array}$ & $\begin{array}{l}\text { VEGF } \\
(\mathrm{ng} / \mathrm{ml})\end{array}$ & $\begin{array}{l}\text { IL-6 } \\
(\mathrm{pg} / \mathrm{ml})\end{array}$ & $\begin{array}{l}\text { CRP } \\
(\mathrm{mg} / \mathrm{l})\end{array}$ \\
\hline HS & 50 & $2.39 \pm 2.53$ & $6.99 \pm 16.64$ & $0.23 \pm 0.29$ & $88.89 \pm 186.88$ & $21.38 \pm 25.98$ \\
\hline IS & 30 & $0.81 \pm 0.78^{\star *}$ & $0.30 \pm 0.31 *$ & $0.10 \pm 0.09 *$ & $10.58 \pm 11.08 *$ & $5.67 \pm 6.52$ ** \\
\hline Control & 20 & $0.05 \pm 0.10^{\star \star}$ & $0.18 \pm 0.29 *$ & $0.01 \pm 0.00 * *$ & $3.43 \pm 1.42^{*}$ & $1.38 \pm 0.69 \star \star$ \\
\hline
\end{tabular}

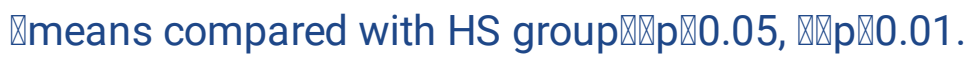

Table 2 The correlation analysis of PTX3 in cerebrospinal fluid

\begin{tabular}{lllll} 
Indicators & PTX3 $(\mathrm{ng} / \mathrm{ml})$ & $\mathrm{HSP70}(\mathrm{ng} / \mathrm{ml})$ & $\mathrm{VEGF}(\mathrm{ng} / \mathrm{ml})$ & $\mathrm{IL}-6(\mathrm{pg} / \mathrm{ml})$ \\
\hline & $r p$ & $r p$ & $r p$ & $r p$ \\
\hline PTX3 & -- & -0.0960 .547 & -0.0780 .625 & $0.631<0.00 \rrbracket$ \\
\hline NIHSS & $0.4120 .007 \rrbracket$ & 0.0420 .793 & -0.860 .590 & $0.608<0.00 \rrbracket$ \\
\hline Barthel & $-0.3510 .023 \rrbracket$ & -0.0190 .907 & 0.0190 .906 & $-0.685<0.00 \rrbracket$
\end{tabular}

๑p\0.05.

Figures 


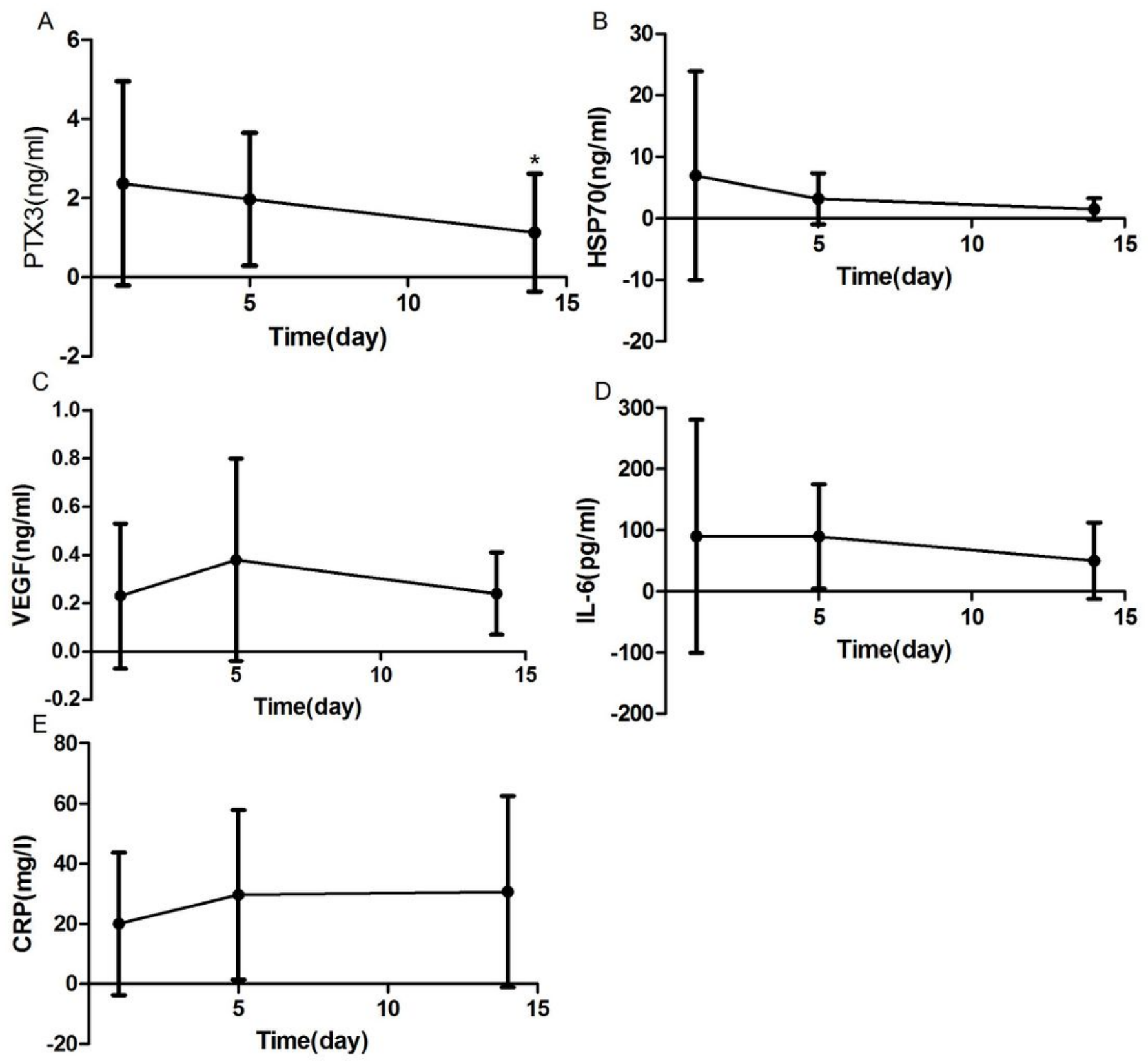

Figure 1

The changes of inflammation and endothelial function in circulating blood in patients with HS A. The change of serum PTX3 level in HS patients, and there was a downward trend on $14 \mathrm{~d}$ after admission. B. The serum HSP70 level in HS patients with no trend change. C. The serum VEGF level in HS patients with no trend change. D. The serum IL-6 level in HS patients with no trend change. $E$. The serum CRP level in HS patients with no trend change. 


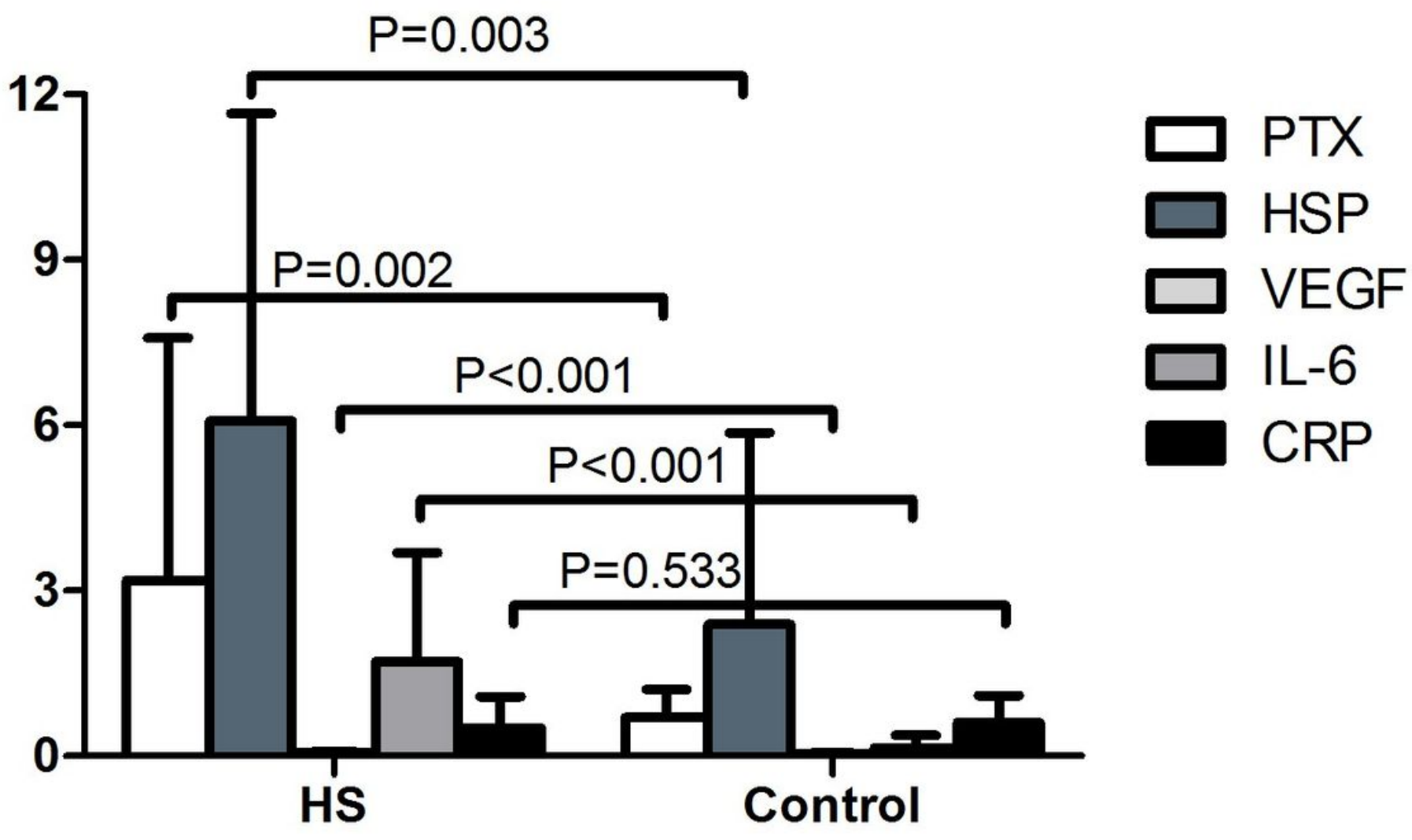

Figure 2

The inflammatory status and endothelial function in brain in HS patients. The levels of PTX3, HSP70, VEGF and IL-6 in cerebrospinal fluid of HS patients were higher than the control subjects, and there was no difference in CRP. 

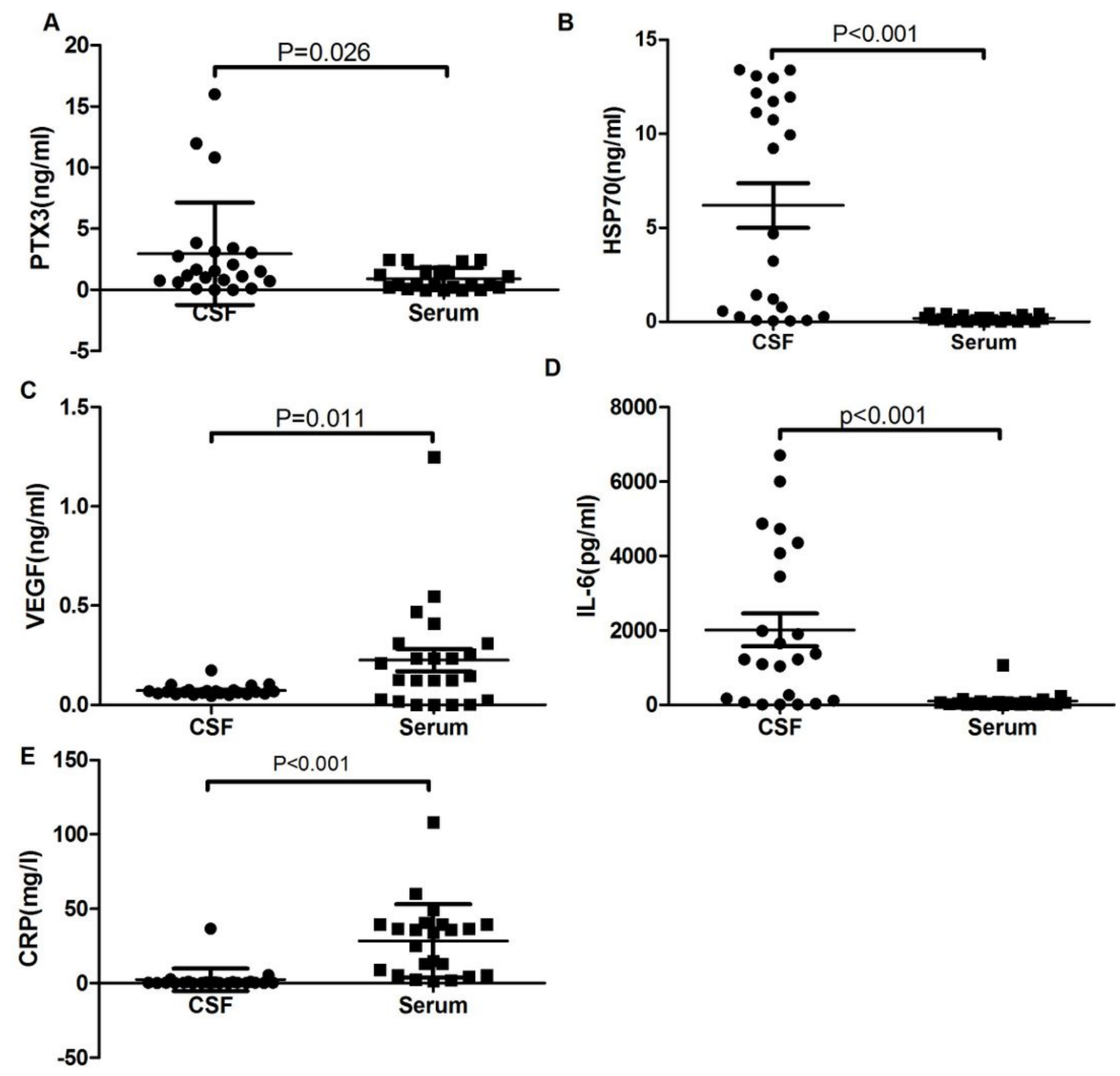

Figure 3

The inflammation and vascular endothelial factors comparison between in neurological system and circulating system in HS patients. A. The cerebrospinal fluid PTX3 level higher than the serum level of PTX3 in HS patients. B. The cerebrospinal fluid HSP70 level higher than the serum level of HSP70 in HS patients. C. The serum VEGF level higher than cerebrospinal fluid VEGF level in HS patients. D. The cerebrospinal fluid IL-6 level higher than the serum level of IL-6 in HS patients. E. The serum CRP level higher than cerebrospinal fluid CRP level in HS patients. 

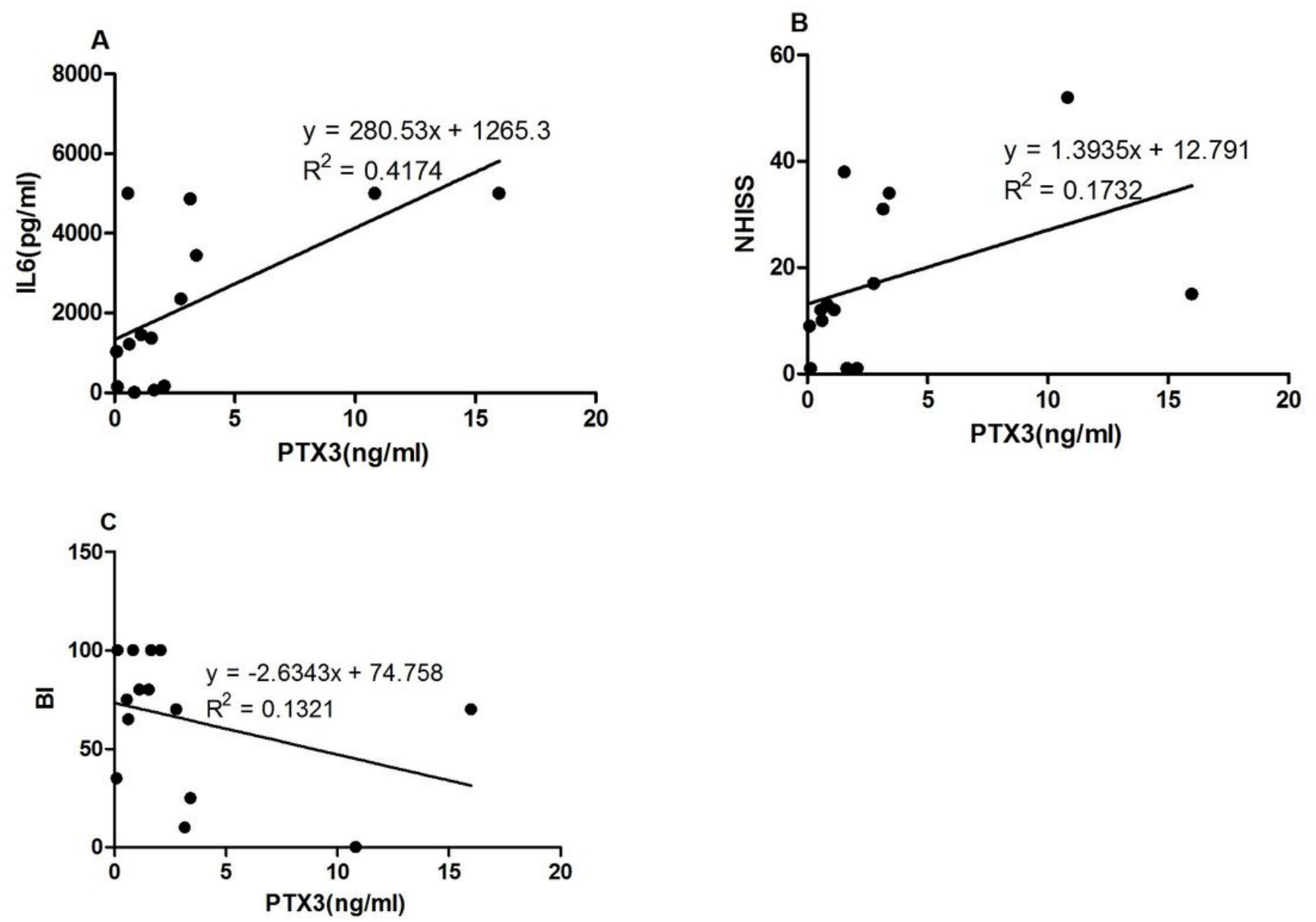

\section{Figure 4}

The correlation analysis of PTX3 in cerebrospinal fluid. A. The cerebrospinal fluid level of PTX3 was positively correlated with IL-6 in patients with HS. B. The cerebrospinal fluid level of PTX3 was positively correlated with NIHSS score in patients with HS. C. The cerebrospinal fluid level of PTX3 was negatively correlated with Barthel index score in patients with HS. 


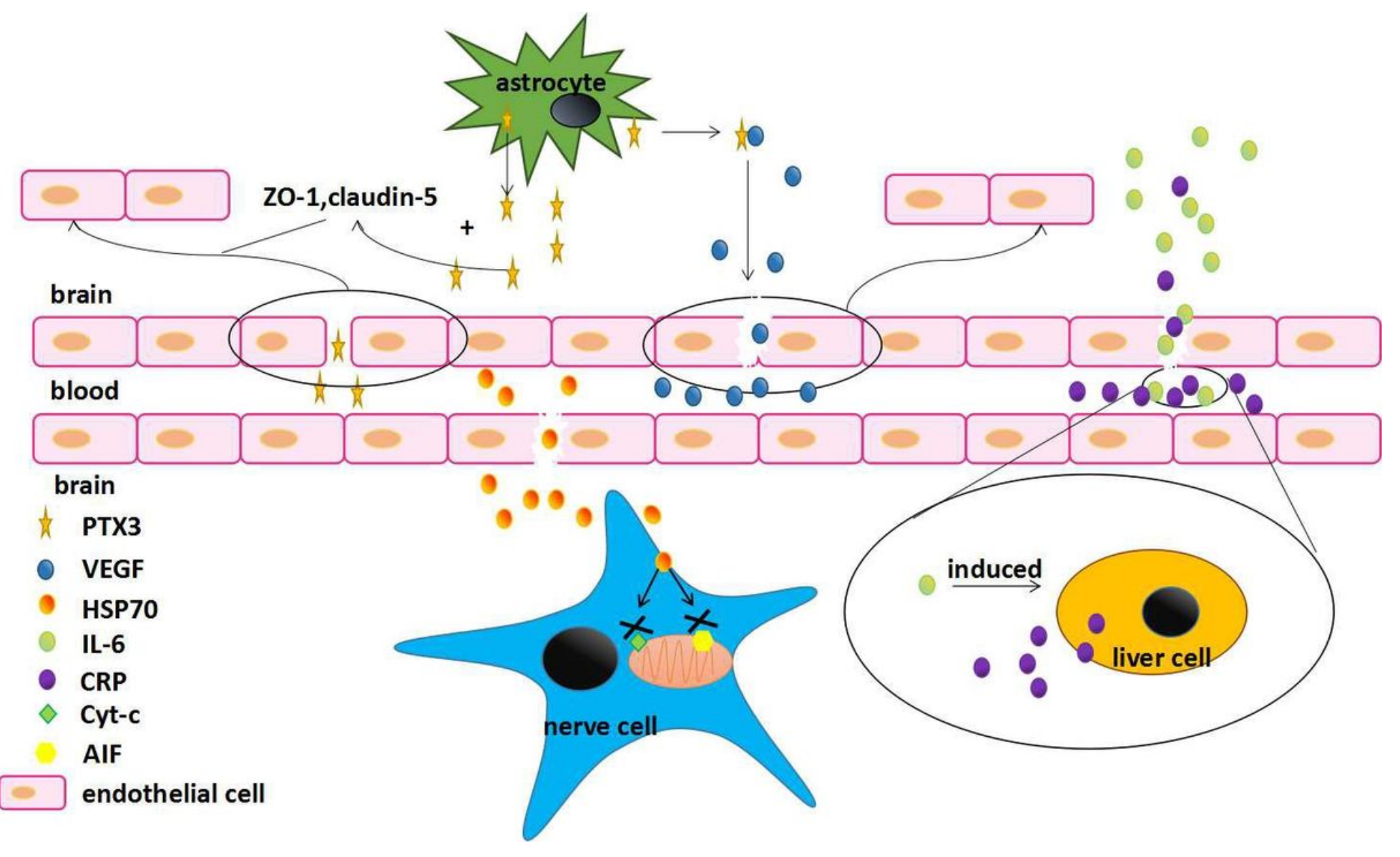

Figure 5

The role of PTX3 in hemorrhagic stroke In patients with HS, the expressions of PTX3, HSP70, VEGF and IL-6 in cerebrospinal fluid were up-regulated on admission, then by respective mechanisms $\otimes$ they reduced neurological injury, broke through the broken BBB into circulating blood, and stimulated the release of various factors, such as $\mathrm{CRP}$, to reduce inflammation and protect the nerve cells. 